\title{
PRESENTACIÓN DE UN CASO DE EMBARAZO ECTOPICO ABDOMINAL A TÉRMINO CON EVOLUCIÓN FAVORABLE MADRE-NIÑO
}

\author{
José Gamboa Barrantes ${ }^{1}$, Félix Dasio Ayala Peralta², Vilma Oros Camargo ${ }^{3}$, \\ Mario Ochoa Rua ${ }^{4}$
}

\begin{abstract}
RESUMEN
El embarazo ectópico abdominal es una entidad extremadamente rara y peligrosa que, de no realizar un diagnóstico adecuado y un tratamiento precoz, lleva a una alta mortalidad materna fetal y donde la posibilidad de llegar a termino son remotas y la supervivencia del feto lo es más aun. En las últimas décadas ha aumentado la frecuencia del embarazo ectópico abdominal que es de 1 por cada 10000. Presentamos el caso de una gestante con embarazo ectópico abdominal a término con feto vivo que llega al servicio de emergencia, Departamento de cuidados críticos del Instituto Nacional Materno Perinatal durante la guardia hospitalaria, la cual es operada y evoluciona favorablemente, tanto la madre como el niño. Se presenta el testimonio gráfico del mismo.
\end{abstract}

Palabras clave: Embarazo abdominal; Embarazo ectópico.

\section{A CASE OF ABDOMINAL ECTOPIC PREGNANCY TO TERM WITH FAVORABLE EVOLUTION MOTHER CHILD}

\begin{abstract}
Abdominal ectopic pregnancy is extremely rare and dangerous, without making a proper diagnosis and early treatment, leads to high maternal fetal and where the possibility of coming to term are remote and fetal survival even more so. In the last decades has increased the frequency of abdominal ectopic pregnancy which is 1 per 10000 . We report the case of a pregnant woman with abdominal ectopic pregnancy to term with live fetus reaching emergency service, Critical Care Department of Maternal and Perinatal Institute during the hospital guard, which is operated and progressing well, both mother and child. We present graphic evidence of it.
\end{abstract}

Key words: Abdominal pregnancy; Ectopic pregnancy

\section{INTRODUCCIÓN}

El embarazo ectópico se estima que ocurre en 1 a 2 por ciento de los embarazos ${ }^{1,2}$. Más del 90 por ciento se localizan en la trompa de Falopio, mientras que el resto del implante en lugares tales como el abdomen, la cicatriz por cesárea (histerotomía), el cuello uterino, y ovario ${ }^{3}$.

La incidencia estimada del embarazo ectópico abdominal es de 1 por cada 10000 nacimientos ${ }^{4}$ y un 1,4 por ciento de los embarazos ectópicos ${ }^{4-6}$. Hay informes de embarazo abdominal que ocurre después de la histerectomía ${ }^{7,8}$.

El embarazo ectópico abdominal está asociado a elevada mortalidad materno fetal. La mortalidad materna es generalmente el resultado de una hemorragia incontrolable, y se ha descrito hasta en el 20 por ciento de los casos. Deformaciones del feto y la muerte perinatal ocurren con más frecuencia que la muerte materna ${ }^{18,19}$.
No se sabe si los embarazos abdominales son una consecuencia de la implantación secundaria de un embarazo tubárico abortado o el resultado de la fertilización intraabdominal del espermatozoide y el óvulo, con la implantación primaria en el abdomen $[5,9]$. Sitios potenciales incluyen el omento, pared pélvica, ligamento ancho, posterior fondo de saco-, los órganos abdominales (por ejemplo, el bazo, el intestino, el hígado), los grandes vasos pélvicos, el diafragma y la serosa uterina ${ }^{5,9-11}$.

Los factores de riesgo para el embarazo abdominal incluyen daño tubario, enfermedad pélvica inflamatoria, endometriosis, técnicas de reproducción asistida, y la multiparidad $^{10,12}$.

Un alto índice de sospecha es importante para hacer un diagnóstico de embarazo abdominal. El hallazgo ecográfico clásico es la ausencia de tejido miometrial entre la vejiga materna y el embarazo ${ }^{9}$. En contraste con los embarazos

\footnotetext{
Médico GínecoObstetra, Jefe del Departamento de Cuidados Críticos del Instituto Nacional Materno Perinatal. Facultad de Medicina Humana " Hipólito Unanue" de la Universidad Nacional Federico Villarreal. Lima, Perú.

2 Médico GinecoObstetra, Magíster en Salud Reproductiva, Facultad de Medicina Humana de la Universidad San Martín de Porres y Facultad de Ciencias de la Salud de la Universidad Norbert Wiener. Director Ejecutivo de la Oficina Ejecutiva de Apoyo a la Investigación y Docencia Especializada-Instituto Nacional Materno Perinatal.

${ }^{3,4}$ Médico GinecoObstetra, Asistente del Departamento de Cuidados Críticos del Instituto Nacional Materno Perinatal.
} 
ectópicos, las mujeres con embarazos abdominales no suelen tener una tendencia anormal en serie los valores de hCG. El diagnóstico diferencial incluye embarazo ectópico en otros lugares, el embarazo intrauterino en un cuerno uterino rudimentario, desprendimiento prematuro de placenta y ruptura uterina.

En cuanto al tratamiento en caso de embarazo abdominal se diagnostica a una edad temprana de gestación (primer trimestre), la cirugía laparoscópica es una opción 5,13,14-15.

En contraste con los embarazos ectópico tubárico, la terapia de metotrexato primaria temprana de gestaciones ha tenido un éxito mínimo ${ }^{16}$. La conducta expectante para ganar madurez fetal se ha intentado y ha tenido éxito en algunos $\operatorname{casos}^{17}$. Si el diagnóstico es tardío se realiza laparotomía la eliminación de la placenta puede conducir a la hemorragia materna en peligro la vida. Se prefiere I ligar el cordón umbilical y la placenta dejando in situ pero se necesitan años para que la masa de la placenta sea absorbida.

Una serie de casos de 10 embarazos abdominales reveló dificultad en el diagnóstico y sólo 6 / 10 fueron diagnosticados de forma preoperatoria. Complicaciones informadas incluyeron hemorragia que requirió transfusión en 9 / 10 las mujeres y la preeclampsia en $3 / 10$; y 5 fetos viables sobrevivieron. Independientemente del lugar de implantación de la placenta un embarazo extrauterino avanzado es una condición seria ${ }^{20}$.

\section{REPORTE DE CASO}

Paciente mujer de 30 años de edad, gestante de 39 semanas por fecha de última regla, con fórmula obstétrica: G3 P2002. Antecedentes: "Marcapaso cardiaco a los 2 años". Primer parto por cesárea por antecedente cardiaco en el año 2000. Segundo parto en el 2003 vía vaginal. Mayor ponderado fetal $3000 \mathrm{~g}$. Control prenatal 10 veces, estuvo hospitalizada en 3 ocasiones previas, a las 14 semanas por amenaza de aborto, enfemedad diarreica

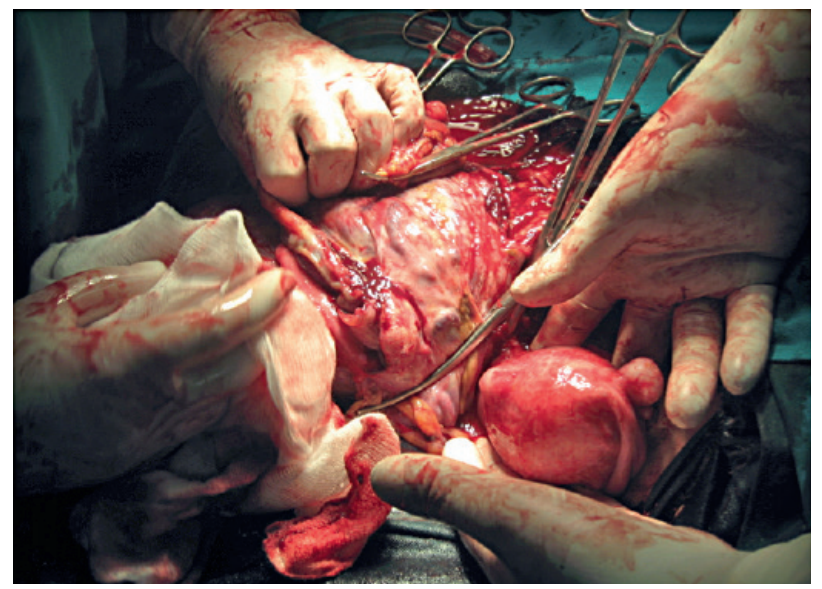

Figura 1. Liberación de placenta y se observa el útero tamaño normal aguda y deshidratación; a las 18 semanas por amenaza de aborto y Traqueítis ; y a las 36 semanas para descartar rotura prematura de membranas y placenta previa centro total no sangrante. Durante la gestación se realizaron 4 ecografías obstétricas.

Ingresa a la institución, el 26 de mayo de 2010, por el servicio de emergencia por presentar dolor abdominal, movimientos fetales presentes y pérdida de tapón mucoso.

Funciones vitales: temperatura $36,6 \mathrm{C}^{\circ}$; frecuencia cardiaca: 72 por minuto; frecuencia respiratoria:17 r/ minuto; presión aterial: 120/80 mmHg. Al exámen obstétrico: altura uterina $30 \mathrm{~cm}$; situación transversa derecha; latido cardíaco fetal de 146 por minuto; no soplos; no DIP; ponderado fetal 3100g; no hidramnios. Tacto vaginal: Dilatación: 0; incorporación: $50 \%$; altura de presentación:-4. Membranas Integras. Pelvis Ginecoide. Sin edemas en miembros inferiores y reflejos normales.

Diagnostico de ingreso: Tercigesta de 39 semanas por fecha de útima regla. Situación transversa. Pródromo de trabajo de parto. Placenta previa total. Cesareada anterior 1 vez. Plan: Cesárea.

Reporte Operatorio: Operación realizada fue laparotomía exploratoria. Extracción fetal y placentaria. Anexectomia izquierda. Bloqueo tubárico derecho.

Procedimiento: Incisión Pfanennstiel en losange. Apertura de cavidad abdominal. Extracción transplacentaria de feto en podálica. Identificación de hallazgos. Ligadura de adherencias de epiplon a placenta. Ligadura del anexo izquierdo en conjunto con ligamento ancho, ambos adheridos a la placenta. Revisión de hemostasia. Revisión de mesenterio, cavidad abdominal y superficie hepática. Ligadura de trompa derecha tipo Pomeroy modificada. Cierre de pared por planos.

Hallazgos: Hemoperitoneo. Feto vivo en cavidad abdominal en situación transversa derecha dorso superior dentro de las membranas amnióticas. Placenta bilobulada

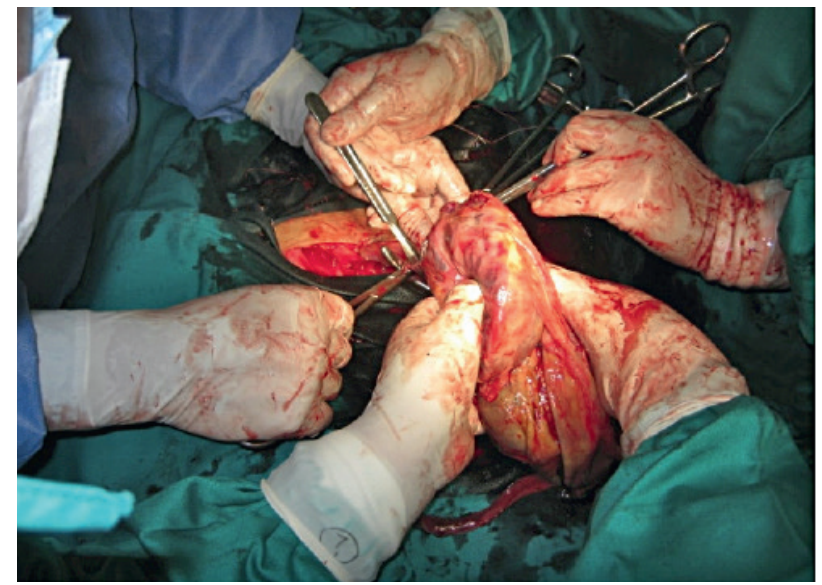

Figura 2. Liberación de la placenta de la cavidad abdominal. 


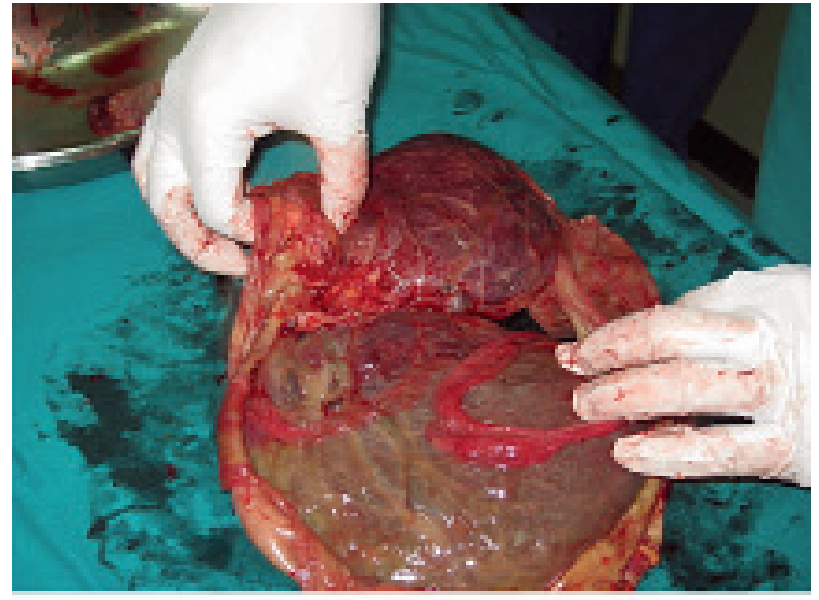

Figura 3. Placenta bilobulada que es liberada de la cavidad abdominal.

adherida a ligamento ancho, trompa y ovario izquierdo también adherida a epiplón (figuras 1, 2 y 3). Útero de aproximadamente 10 centímetros con mioma subseroso en cara anterior de $\pm 2 \mathrm{~cm}$ (figura 1). Trompa y ovario derecho normal. Recien nacido vivo con Apgar 9 al minuto y a los 5 minutos, de sexo femenino, cuyo peso fue de 4002 gramos y $50 \mathrm{~cm}$ de talla (figura 4). Presencia de escaso meconio en cavidad abdominal. Sangrado intraoperatorio: $2000 \mathrm{cc}$.

Diagnóstico postoperatorio: Embarazo ectópico abdominal. Hemoperitoneo. Miomatosis uterina.

Manejo postoperatorio: Se realiza en la Unidad de Cuidados Intensivos Maternos. Recibió transfusión sanguínea, hidratación, antibioticoterapia y monitoreo hemodinámico materno con evolución favorable, saliendo de alta médica del hospital al cuarto día postoperatorio.

\section{DISCUSIÓN}

Este caso ha sido de difícil diagnóstico, a pesar que tuvo 10 controles prenatales institucionales, se le hospitalizó en 3 oportunidades previas y se le realizaron 4 ecografías obstétricas, nunca se sospecho de un embarazo ectópico abdominal, siendo manejada como un embarazo intrauterino de alto riesgo, por el antecedente del marcapaso cardiaco, la cesárea anterior y la supuesta "placenta previa centro total" por ecografía.

En realidad el embarazo ectópico abdominal es bien raro y de muy difícil diagnostico sino se sospecha en él, y que llegue a término con feto vivo es más raro aún, ya que la mayoría de los fetos mueren tempranamente y muchos casos sólo son diagnosticados durante el acto operatorio.

Estos casos representan alto riesgo de mortalidad materna por la hemorragia intraabdominal súbita que pueden presentar, la cual se desencadena muchas veces

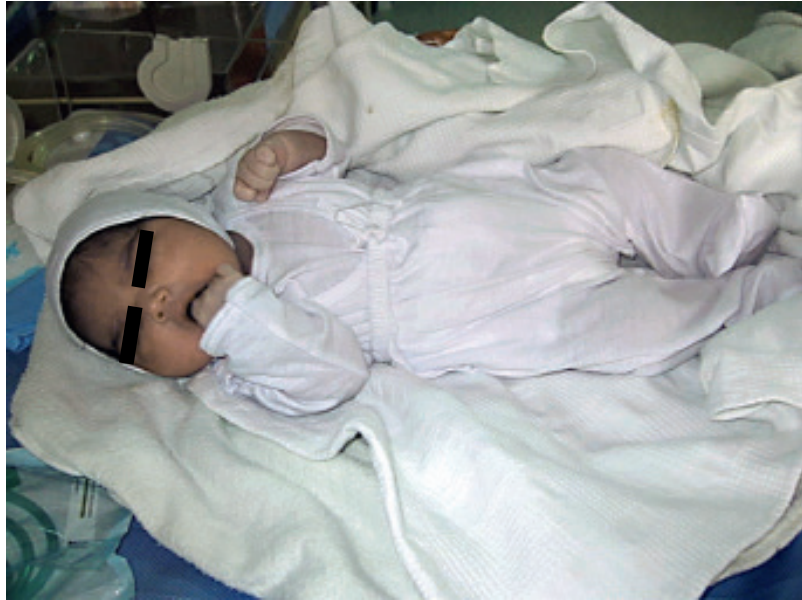

Figura 4. Recien nacida a término en buenas condiciones.

intra operatoriamente, en el momento de extracción de la placenta.

Hay que tener presente que cuando el diagnóstico se realiza antes de las 24 semanas de gestación el tratamiento de elección es quirúrgico (laparotomía exploratoria) por la imposibilidad de supervivencia fetal; en cambio, cuando el diagnostico se realiza después de las 24 semanas de gestación se recomienda un tratamiento intrahospitalario conservador en expectación armada, esperando la viabilidad fetal.

En este caso se hizo, como la mayoría de autores preconizan, la extracción placentaria intraoperatoria, ya que no se encontraba firmemente adherida a ningún órgano vital, sino estaba adherida sobre el epiplon, el ligamento ancho y parte del anexo izquierdo, de lo contrario se debió seccionar el cordón umbilical lo más cerca posible de la placenta y dejarla en su lecho de inserción, y luego realizar seguimiento ecográfico y análisis seriados de gonadotrofina coriónica para determinar su evolución y utilizar también metotrexate para ayudar a la reabsorción de la placenta.

Se debe destacar el poco tiempo de hospitalización postoperatoria de la paciente, solo un día en UCIM y 3 días en hospitalización para salir de alta en buenas condiciones. Es importante también resaltar la evolución del feto que llego a tener un peso de 4,002 gramos y nacer con un Apgar de 9 al minuto y a los 5 minutos, con una evolución favorable.

En nuestro medio en una publicación reciente Falcón ${ }^{21}$ reporta sobre un caso de gestación abdominal con feto vivo de 34 semanas que nació con 1880 gramos y que posteriormente falleciera a los 17 días de nacido por sepsis neonatal, a comparación de nuestro caso que la madre salió de alta con su recién nacida en buenas condiciones. 


\section{REFERENCIAS BIBLIOGRÁFICAS}

1. Pregnancy-Related Mortality Ratios, United States 19911999. CDC. MMWR Vol 52, ss-2, February 21, 2003. Disponible en http://www.uptodate.com/contents/abdominalpregnancy-cesarean-scar-pregnancy-and-heterotopicpregnancy

2. Centers for Disease Control and Prevention (CDC). Ectopic pregnancy--United States,1990-1992. MMWR Morb Mortal Wkly Rep 1995; 44:46.

3. Bouyer, J, Coste, J, Fernández, H, et al. Sites of ectopic pregnancy: a 10 year population-based study of 1800 cases. Hum Reprod 2002; 17:3224.

4. Atrash, HK, Friede, A, Hogue, CJ. Abdominal pregnancy in the United States: frequency and maternal mortality. Obstet Gynecol 1987; 69:333.

5. Dover, RW, Powell, MC. Management of a primary abdominal pregnancy. Am J Obstet Gynecol 1995; 172:1603.

6. Fisch, B, Peled, Y, Kaplan, B, et al. Abdominal pregnancy following in vitro fertilization in a patient with previous bilateral salpingectomy. Obstet Gynecol 1996; 88:642.

7. Binder, DS. Thirteen-week abdominal pregnancy after hysterectomy. J Emerg Med 2003; 25:159.

8. Fader, AN, Mansuria, S, Guido, RS, Wiesenfeld, HC. A 14-week abdominal pregnancy after total abdominal hysterectomy. Obstet Gynecol 2007; 109:519.

9. Varma, R, Mascarenhas, L, James, D. Successful outcome of advanced abdominal pregnancy with exclusive omental insertion. Ultrasound Obstet Gynecol 2003; 21:192.

10. Ludwig, M, Kaisi, M, Bauer, O, Diedrich, K. The forgotten child--a case of heterotopic, intra-abdominal and intrauterine pregnancy carried to term. Hum Reprod 1999; 14:1372.
11. Fishman, DA, Padilla, LA, Joob, A, Lurain, JR. Ectopic pregnancy causing hemothorax managed by thoracoscopy and actinomycin D. Obstet Gynecol 1998; 91:837.

12. Tsudo, T, Harada, T, Yoshioka, H, Terakawa, N. Laparoscopic management of early primary abdominal pregnancy. Obstet Gynecol 1997; 90:687.

13. Morita, Y, Tsutsumi, O, Kuramochi, K, et al. Successful laparoscopic management of primary abdominal pregnancy. Hum Reprod 1996; 11:2546.

14. Pisarska, MD, Casson, PR, Moise, KJ Jr, et al. Heterotopic abdominal pregnancy treated at laparoscopy. Fertil Steril 1998; 70:159.

15. Gerli, S, Rossetti, D, Baiocchi, G, et al. Early ultrasonographic diagnosis and laparoscopic treatment of abdominal pregnancy. Eur J Obstet Gynecol Reprod Biol 2004; 113:103.

16. Zinger, M, Rosenfeld, D. Failed treatment of abdominal pregnancy with methotrexate. A case report. J Reprod Med 2001; 46:392.

17. Beddock, R, Naepels, P, Gondry, C, et al. Diagnosis and current concepts of management of advanced abdominal pregnancy. Gynecol Obstet Fertil 2004; 32:55.

18. Ayinde, OA, Aimakhu, CO, Adeyanju, OA, Omigbodun, AO. Abdominal pregnancy at the University College Hospital, Ibadan: a ten-year review. Afr J Reprod Health 2005; 9:123.

19. Stevens, CA. Malformations and deformations in abdominal pregnancy. Am J Med Genet 1993; 47:1189.

20. Worley, KC, Hnat, MD, Cunningham, FG. Advanced extrauterine pregnancy: diagnostic and therapeutic challenges. Am J Obstet Gynecol 2008; 198:297.

21. Falcón AR. Gestación abdominal con feto vivo. Rev Per Ginecol Obstet 2009; 55: 281-284. 